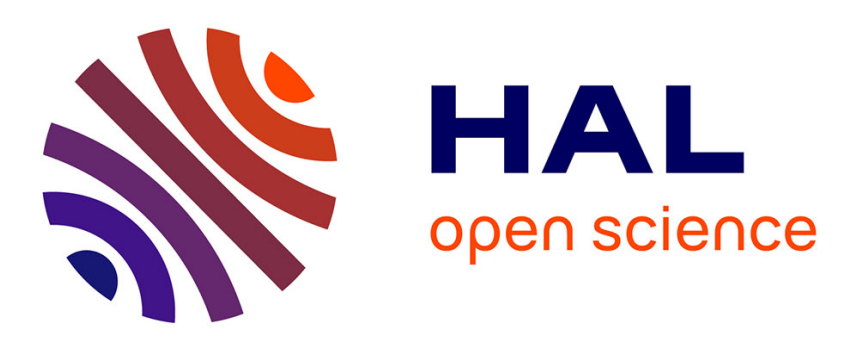

\title{
Comparison of Analysis Line and Polytopes Methods to Determine the Result of a Tolerance Chain
}

\author{
Laurent Pierre, Bernard Anselmetti
}

\section{To cite this version:}

Laurent Pierre, Bernard Anselmetti. Comparison of Analysis Line and Polytopes Methods to Determine the Result of a Tolerance Chain. Journal of Computing and Information Science in Engineering, 2015, 10.1115/1.4029049 . hal-01239248

\section{HAL Id: hal-01239248 \\ https://hal.science/hal-01239248}

Submitted on 7 Dec 2015

HAL is a multi-disciplinary open access archive for the deposit and dissemination of scientific research documents, whether they are published or not. The documents may come from teaching and research institutions in France or abroad, or from public or private research centers.
L'archive ouverte pluridisciplinaire HAL, est destinée au dépôt et à la diffusion de documents scientifiques de niveau recherche, publiés ou non, émanant des établissements d'enseignement et de recherche français ou étrangers, des laboratoires publics ou privés. 


\title{
Comparison of analysis line and polytopes methods to determine the result of a tolerance chain
}

\author{
PIERRE, Laurent ${ }^{1}$ \\ LURPA, ENS Cachan, Univ Paris-Sud, F-94235 Cachan, France \\ 61, av. du Président Wilson,94235 Cachan Cedex - France \\ laurent.pierre@lurpa.ens-cachan.fr \\ ANSELMETTI, Bernard \\ LURPA, ENS Cachan, Univ Paris-Sud, F-94235 Cachan, France \\ 61, av. du Président Wilson, 94235 Cachan Cedex - France \\ Bernard.anselmetti@lurpa.ens-cachan.fr
}

\begin{abstract}
Functional tolerancing must ensure the assembly and the functioning of a mechanism. This paper compares two methods of tolerance analysis of a mechanical system: the method of "analysis lines" and the method of "polytopes".

The first method needs a discretization of the ending functional surface according to various analysis lines placed on the outer-bound of the face and oriented along the normal of the surface. The second method uses polytopes. The polytopes are defined from the acceptable limits of the geometric deviations of parts and possible displacements between two parts. Minkowski sums and intersections polytopes are then carried out to take into account all geometric variations of a mechanism.
\end{abstract}

\section{KEYWORDS}

Functional tolerancing; tolerance analysis; Analysis line; Polytope; ISO standard of tolerancing

\footnotetext{
${ }^{1}$ Corresponding author information can be added as a footnote.
} 
Journal of Computing and Information Science in Engineering

\section{INTRODUCTION}

\subsection{Functional tolerancing context}

Now the evolution of digital tools allows designers to define their entire mechanism in a CAD environment. The parts are described as perfect shape with nominal dimensions. To ensure interchangeability and allow the production of parts at lower cost, it is very important to allocate the widest possible tolerances, while ensuring that the functional requirements of the mechanism are respected. With this approach, the designer chooses the geometric specifications and tolerances. He must ensure that the combination of the variations permitted by the tolerances will be compatible with each requirement. This step is called tolerance analysis.

\subsection{State of art}

Tolerancing process consists in specification synthesis, tolerancing analysis, tolerancing synthesis and tolerancing verification [1-3].

The specification synthesis determines the set of functional specifications to impose on parts with respect to a functional requirement $[4,5]$.

Traditionally, tolerance analysis is provided by a tolerance chart model in only one direction which is simply to sum of tolerances of influential parts. Soon as there is an angular effect in the chain of dimensions, designers are still quite poor. Some analytics methods calculates the result for each requirement using analytical tolerance chains [6-8] or a statistic methods [9-11] and Monte Carlo methods $[12,13]$.

The variational approach of geometric tolerancing differs from parametric approaches [14]. The variational approach oftolerancing consists of characterising the 
Journal of Computing and Information Science in Engineering

relative position of two surfaces from two different parts of a system by intersections and Minkowski sums of sets of geometric constraints defined by half-spaces. The domains [15] and T-Maps [16] manipulate half-spaces of which the boundaries are generally not linear. In contrast, other methods use half-spaces of which the boundaries are linear like polyhedral objects [17] and polytopes [18, 19].

Indeed, there are very few commercial software tools for the analysis of tolerances in 3D. The principle is based on the simulation of assemblies:

- Mécamaster [20] simulates the assembly of nominal parts and introduces deviations between parts corresponding to tolerances of the support surfaces or clearance in joint.

- Cetol [21], 3DCS [22] generate, by Monte Carlo methods, parts with position, orientation or form defects corresponding to the ISO specifications. Such software measures the characteristic of the requirement on the set of assemblies obtained with simulated parts.

- Anatole [23] characterizes defects of surfaces with small displacement torsor. The equations expressing the displacement of the parts relative to their nominal positions are based on contact between parts to give a formal relationship of part tolerances.

Few scientific studies have been devoted to the allocation of tolerance. Some purely mathematical approaches have been derived, and these essentially develop cost functions to varying levels of sophistication [24-26].

Functional tolerancing must be including in product life management [27]. 
Journal of Computing and Information Science in Engineering

\subsection{Position of proposed work}

Since 1997, B. Anselmetti developed the CLIC method (a French acronym for "Localization Tolerancing with Contact Influence") [5]. This system proposes the automatic generation of functional requirements and functional specifications in accordance with ISO tolerancing standards. The result of each tolerance chart is described by an EXCEL formula according to tolerances of influential parts. This structure allows optimizing the nominal dimensions of CAD model to maximize tolerances [26].

In this software, tolerance analysis is ensured by the analysis line method. The ending functional surface is discretized by various analysis lines placed on the outerbound of the face and oriented along the normal to the surface. The principle consists in the sum of the influence of defects in each junction on analysis line using relationship pre-established for all classical types of junctions. The calculation is very fast and expresses the result with a formula function of tolerances with worst case or with a statistical approach [28]. The method of operations on polytopes in tolerance analysis is a variational approach based on the work initiated by Flemming in 1988. It is based on the operations of sets of geometric constraints [29]. The set of possible positions of a surface within a tolerance zone is characterized by a set of geometric constraints. This allows characterizing the geometric variations of a part according to orientation or position ISO specifications [15-17, 30]. Similarly, a set of geometric constraints characterises all relative positions between two distinct surfaces potentially in contact [31]. Fleming established the correlation between the accumulated defects limits on 
Journal of Computing and Information Science in Engineering

parts and the Minkowski sum of finite sets of geometric constraints [29]. A summary of these issues is detailed in [32]. Giordano showed that the relative position between two parts in contact resulting from a number of potential contacts can be formalized by an intersection operation of a set of geometric constraints [33]. More generally, the variational tolerancing approach characterises the relative position between two surfaces of two parts of any system by intersections and Minkowski sums of sets of geometric constraints. This sets of constraints result from ISO specifications expressed in parts and contact characteristics between two parts [34]. Minkowski sum algorithms applied to the problem of tolerance analysis have been developed [35 36]. A variational method of tolerance analysis using the method based on operations on polytopes is proposed by $[18,19]$. This method was developed to take into account thermomechanical strains.

The aim of this paper is to compare these two methods on an example to show the similarities and limitations.

\section{Definition of requirement}

\subsection{Mechanism description}

The mechanism is composed of two rigid parts with two bores (Fig. 1). The shaft is represented by a single cylinder which is mounted with clearance in a bore of the cover and in a bore of the housing. The cover is assembled and screwed onto the housing, before introducing the shaft. It must therefore be ensured that the shaft can be fitted in the mechanism in any case. 
Journal of Computing and Information Science in Engineering

\subsection{Functional tolerancing}

Functional tolerancing was performed with the Quick GPS software developed by

B. Anselmetti [37] in the CATIA environment with Functional Tolerancing Annotation Workshop.

The junction is described by a positioning table of the cover on the housing (Fig. 2).

The housing is the base of this mechanism. The cover is setting-up on the housing by a primary plane and two bores which receive two pins blocked in the housing. There is clearance between the pins and the cover.

A simple matrix shows all the links in the part studied (Fig. 3). The hole of the cover has to be positioned relative to the junction with the housing.

These data are sufficient for the QUICK GPS software that automatically generates annotations in the 3D CAD model (Fig. 4). The designer can adjust the tolerances.

Each part has a main datum reference frame $A B$ and a location of the bore.

\subsection{Functional requirement}

This paper examines more specifically the assembling of the shaft in the sub system composed by housing and cover. The assembling is difficult when the shaft and the bores are at maximum material condition, when the cover and housing are shifted due to the clearance between the pins and the cover (Fig. 5). 
Journal of Computing and Information Science in Engineering

The shift is maximal when the distances between the pins are the same on both parts and the pins and the holes in the cover are at minimum material condition with a maximum clearance of 0.04 .

The problem is really three-dimensional, because the holes are not in the plane containing the two pins. But to simplify the Fig. 5, the pins are shown in the plane of bores.

\subsection{Methodology}

At maximum material condition, the shaft is considered a perfect cylinder of diameter 29.96. The envelop diameters of the bores are $29.98 \mathrm{~mm}$. If the straightness in common zone of the two holes is $r$, then the free space for the shaft is $29.98-r$. The straightness has to be less than 0.02 in all cases.

\subsection{Tolerancing and significant dimensions}

Fig. 1 depicts the tolerancing corresponding to straightness requirement.

\section{Analysis line method}

\subsection{Principle}

The analysis lines method was developed in 2004 , to calculate the resulting 3D with a statistical approach. The challenge is to globalize the influence dues to translation and angular deviations, whatever the tolerance values. Calculation equations show that the accumulation occurs naturally in certain points and in particular directions called analysis points and analysis directions.

The analysis line method is based on transfer relations that have been established for ten classical junctions (Primary plane / secondary plane / tertiary plane, 
Journal of Computing and Information Science in Engineering

Primary plane / secondary cylinder / tertiary, Primary plane / 2 secondary holes, Primary plane / n secondary holes; Primary cylinder / secondary plane / tertiary, straightness or flatness in common zone ...).

The linear relationship gives the influence of a given junction in the worst case, directly on the functional requirement. This requires a discretization and successive studies in a limited number of directions. The advantage of this method is that for each study, the analysis direction is known. It is very easy to define the position of the part in the worst case permitted by clearance in the junction.

Other junctions can often be modelled by 6 points of contact with a linear relationship. Some complex connections cannot be treated directly. The solution proposed by Robin Chavanne [38] is to use a solver to find the worst case situation and to find the 6 contact points to establish the linear relationship.

\subsection{Transfer of straightness}

The requirement studied thus reduces to straightness between two coaxial bores. The deviation from straightness $r$ is the diameter of a cylinder containing both real axis of the two bores.

The problem is three-dimensional. The analysis line method proposes to study in 8 radial directions $\mathrm{f} 1$ to $\mathrm{f} 8$.

Fig. 8 can be applied to all cases of straightness in common zone of two coaxial cylinders (Fig. 8a).

A location and an orientation specifications are placed on each bore with respect to a common datum reference frame fixed on the housing (Fig. 8b). 
Journal of Computing and Information Science in Engineering

Fig. $8 \mathrm{c}$ shows that in each plane in fi direction, straightness is proportional to the sum of the maximum displacement $d\left(C_{H}, \mathbf{f i}\right)$ of point $C_{H}$ of the housing axis in $\mathbf{f i}$ direction and the maximal displacement $d\left(C_{c},-\mathbf{f i}\right)$ of point $C_{c}$ of the cover axis projected in point $C$ in $-\mathbf{f i}$ direction. Lengths of bores are respectively $E_{C}$ and $E_{H}$. The distance between the bores is $L_{B C}$. The symmetrical condition is presented in Fig. 8d.

The relationship of transfer is therefore:

$$
r_{i}=\max \left\{\begin{array}{l}
\frac{E_{C}}{E_{C}+L_{B C}}\left[d\left(C_{H}, \mathbf{f i}\right)+d\left(C_{C},-\mathbf{f i}\right)\right] ; \\
\frac{E_{H}}{E_{H}+L_{B C}}\left[d\left(B_{H}, \mathbf{f i}\right)+d\left(B_{C},-\mathbf{f i}\right)\right]
\end{array}\right\}
$$

These displacements $d\left(B_{H}, \mathbf{f i}\right), d\left(C_{H}, \mathbf{f i}\right), d\left(B_{C},-\mathbf{f i}\right) ; d\left(C_{C},-\mathbf{f i}\right)$ should be calculated relative to the same reference. The reference is chosen on the median line of both axis $B$ of the datum reference frame $A B$ of the housing.

Firstly, the maximal displacements of the points $B_{C}$ and $C_{C}$ of the cover axis are calculated with respect to the nominal cover axis according to the specifications of the bore.

The nominal cover axis is also the median line of both reference axis $B$ of the cover

In the worst case, point $B_{C}$ moves in $B_{C}^{\prime}$. The displacement of point $B_{C}$ is limited by the location tolerance $t_{3}$ :

$d\left(B_{C}, \mathbf{f i}\right)=\mathrm{t}_{3} / 2$ 
Point $C_{c}$ moves in $C_{c}^{\prime}$. The inclination of the axis of the bore is limited by the specification of orientation $t_{4}$. The displacement of point $C_{C}$ is:

$d\left(C_{C}, \mathbf{f i}\right)=\mathrm{t}_{3} / 2+\mathrm{t}_{4} \cdot\left(\mathrm{L}_{\mathrm{OB}}+\mathrm{L}_{\mathrm{OC}}\right) / \mathrm{E}_{\mathrm{C}}$

The calculation is similar to the points $B_{H}$ and $C_{H}$ of the housing axis with the tolerance $t_{1}$ in location and $t_{2}$ in orientation.

$d\left(C_{H}, \mathbf{f i}\right)=\mathrm{t}_{1} / 2$
$d\left(B_{H}, \mathbf{f i}\right)=\mathrm{t}_{1} / 2+\mathrm{t}_{2} \cdot \mathrm{L}_{\mathrm{BC}} / \mathrm{E}_{\mathrm{H}}$

By symmetry, these relationships are independent of analysis directions fi.

Displacements of points $B$ and $C$ of the nominal cover axis must now be determined according to the housing.

\subsection{Influence of clearance in junction housing/cover}

The problem is to calculate the displacements of the nominal axis of cover in $B_{c}$ and in $C_{C}$

The cover is setting-up on the housing with a primary plane and two pins. The datum reference frame of the housing is $A B$. Datum plane $A$ of the housing and datum plane A of the cover are supposedly confused.

The nominal axis of the holes being perpendicular to the primary plane, there is no angular deviations between both nominal axis of the housing and cover.

The shift of the nominal axis is due only to the mobility permitted by the clearance $\mathrm{J}$ between the housing and the cover. Fig. 10 shows both axis $\mathrm{E}$ and $\mathrm{F}$ of the housing holes, and both axis $\mathrm{E}^{\prime}$ and $\mathrm{F}^{\prime}$ of the cover holes in the cover. 
Journal of Computing and Information Science in Engineering

The connection between these bores is provided by the pins blocked in the housing. Displacement is maximal when the distances between the holes are identical in housing and the cover and when the pins and the cover holes are at least material condition with a maximum clearance $\mathrm{J}$.

If the analysis line $d\left(B_{c}, \mathbf{f i}\right)$ cut segment $E F$, the maximum displacement in the direction fi is equal to $\mathrm{J} / 2$.

If the analysis line $d\left(B_{C}, \mathbf{f i}\right)$ does not cut the segment $E F$, the displacement of $B_{C}$ is maximal when $\mathrm{E}$ move in $\mathrm{E}^{\prime}$ and when $\mathrm{F}$ move in $\mathrm{F}^{\prime}$ in $\varphi$ direction.

The displacement of the point $B_{C}$ depends of the angle $\varphi$. The derivation shows that the displacement is maximum, irrespective of the angle $\theta$ value, when the points $B_{C}, E$ and $E^{\prime}$ are aligned, either for $\varphi=\arctan \left(O B_{C} / O E\right)$.

In this case, the displacement of $B_{C}$ is $\mathrm{J} / \cos \varphi \cdot \mathbf{y}$. The maximal displacement in $\mathbf{f i}$ direction is:

$$
d\left(B_{c}, \mathbf{f i}\right)=J \cdot \cos \theta / \cos \varphi
$$

The maximal clearance between pins and hole of cover is:

$$
J=D_{\text {max_hole }}-D_{\text {min_pin }}=t_{5}+t_{6}
$$

Both distances $L_{E F}=100 \mathrm{~mm}$ and $L_{O B}=60 \mathrm{~mm}, 1 / \cos \varphi=1.56$. Calculated displacements are:

For $\mathbf{f 1}=\mathbf{x}$ and $\mathbf{f} \mathbf{5}=-\mathbf{x}$ : 
$d\left(B_{C}, \mathbf{f i}\right)=J / 2$

For $\mathbf{f 3}$ and $\mathbf{f 7}, \cos \theta= \pm 1$ :

$d\left(B_{c}, \mathbf{f i}\right)=J /(2 \cdot \cos \varphi)=1.56 \cdot J / 2$

For $\mathbf{f 2}, \mathbf{f 4}, \mathbf{f 6}$ and $\mathbf{f 8}, \theta=\pi / 4$ :

$d\left(B_{c}, \mathbf{f i}\right)=J /(2 \cdot \sqrt{2} \cdot \cos \varphi)=1.104 \cdot J / 2$

\subsection{Full condition}

The full condition giving the straightness in the worst case is the maximum value of 8 relationships in 8 directions $\mathbf{f i}$.

$r_{i}=\max \left\{\begin{array}{l}\frac{E_{C}}{E_{C}+L_{B C}}\left[d\left(C_{H}, \mathbf{f i}\right)+d\left(C_{C},-\mathbf{f i}\right)\right] ; \\ \frac{E_{H}}{E_{H}+L_{B C}}\left[d\left(B_{H}, \mathbf{f i}\right)+d\left(B_{C},-\mathbf{f i}\right)\right]\end{array}\right\}$

With

$d\left(C_{H},-\mathbf{f i}\right)=\mathrm{t}_{1} / 2$

$d\left(B_{H},-f i\right)=t_{1} / 2+t_{2} \cdot L_{B C} / E_{H}$

Maximal displacements of $B_{C}$ and $C_{c}$ depend on fi direction. The displacement is maximal for $\mathbf{f} \mathbf{3}$ and $\mathbf{f 7}$ directions in plane $(\mathbf{y}, \mathbf{z})$ :

$$
\begin{aligned}
& d\left(C_{C}, \mathbf{f i}\right)=\mathrm{t}_{3} / 2+\mathrm{t}_{4} \cdot \mathrm{L}_{B C} / \mathrm{E}_{\mathrm{C}}+\left(\mathrm{t}_{5}+\mathrm{t}_{6}\right) /(2 \cdot \cos \varphi) \\
& d\left(B_{C}, \mathbf{f i}\right)=\mathrm{t}_{3} / 2+\left(\mathrm{t}_{5}+\mathrm{t}_{6}\right) /(2 \cdot \cos \varphi)
\end{aligned}
$$

The straightness is obtained in plane $(\mathbf{y}, \mathbf{z})$ : 


$$
r_{i}=\max \left\{\begin{array}{l}
\frac{E_{C}}{E_{C}+L_{B C}}\left[\frac{\mathrm{t}_{1}}{2}+\frac{\mathrm{t}_{3}}{2}+\mathrm{t}_{4} \cdot \frac{\mathrm{L}_{\mathrm{BC}}}{\mathrm{E}_{C}}+\frac{\mathrm{t}_{5}+\mathrm{t}_{6}}{2 \cdot \cos \varphi}\right] \\
\frac{E_{H}}{E_{H}+L_{B C}}\left[\frac{\mathrm{t}_{1}}{2}+\frac{\mathrm{t}_{3}}{2}+\mathrm{t}_{2} \cdot \frac{\mathrm{L}_{\mathrm{BC}}}{\mathrm{E}_{\mathrm{H}}}+\frac{\mathrm{t}_{5}+\mathrm{t}_{6}}{2 \cdot \cos \varphi}\right]
\end{array}\right\}
$$

with: $\varphi=\arctan \left(O B_{c} / O E\right)$

These relationships are linear and simple to integrate into a system of equations for the synthesis of tolerances.

\section{Method by operations on polytopes}

\subsection{Transfer of geometric deviations on parts}

A real surface resulting from the manufacturing is modelled by a surface of substitution that has the same type as the nominal surface. The deviations between the surface of substitution and nominal surface are called geometric deviations. The surface of substitution $i$ of the part $j$ is noted $i, j$. Geometric deviations between a surface of substitution 1,1 and a surface of substitution 1,2 are formalized by a small displacement torsor $\left[d_{1,1 / 1,2}\right]$, where the vector $\boldsymbol{\rho}_{1,1 / 1,2}$ is the rotation vector of 1,1 with respect to 1,2 while the vector $\boldsymbol{\varepsilon}_{\mathrm{B}-1,1 / 1,2}$ is the translational vector of 1,1 with respect to 1,2 at point $B$ :

$$
\left[d_{1,1 / 1,2}\right]=\left[\begin{array}{c}
\boldsymbol{\rho}_{1,1 / 1,2} \\
\boldsymbol{\varepsilon}_{\mathrm{B}-1,1 / 1,2}
\end{array}\right]_{\mathrm{B}}
$$

According to the specification of the location of axis 1,1 of the bore of the housing 1 (Fig. 6), the axis of the cylindrical surface of substitution 1,1 is within a tolerance zone $Z T . Z T$ is a cylinder of diameter $\varnothing t_{1}$ orthogonal to the plane $A$ and 
Journal of Computing and Information Science in Engineering

centred on nominal axis relating to $A B$ datum reference frame. To ensure that the axis 1,1 of the surface is located in the zone of tolerance, it is necessary to write, to both points $C$ and $D$, the following relationship where $\mathbf{n}_{\theta i}$ is a unitary vector orthogonal to the axis $\mathbf{z}$ and $\theta i$ is the discretization angle around the axis $\mathbf{z}$ :

$$
\begin{aligned}
& \left\{\begin{array}{l}
-\frac{t_{1}}{2} \leq \boldsymbol{\varepsilon}_{C-1,1 / A B} \cdot \mathbf{n}_{\theta i} \leq \frac{t_{1}}{2} \\
-\frac{t_{1}}{2} \leq \boldsymbol{\varepsilon}_{D-1,1 / A B} \cdot \mathbf{n}_{\theta i} \leq \frac{t_{1}}{2}
\end{array}\right\} \\
& \text { with }\left\{\begin{array}{l}
\mathbf{n}_{\theta i}=\cos \theta_{i} \cdot \mathbf{x}+\sin \theta_{i} \cdot \mathbf{y} \\
\theta_{i}=i \frac{\pi}{n}, 0 \leq i<n \text { and }(i, n) \in \mathbb{N}
\end{array}\right.
\end{aligned}
$$

The relation (15) is expressed according to the rotation vector and the translation vector of the deviation of the point $\mathrm{P}$ middle of the bounded by line segment B and C (Fig. 5):

$$
\begin{aligned}
& \left\{\begin{array}{l}
-\frac{t_{1}}{2} \leq\left(\boldsymbol{\varepsilon}_{\mathrm{p}-1,1 / A B}+\mathbf{C P} \wedge \boldsymbol{\rho}_{1,1 / A B}\right) \cdot \mathbf{n}_{\theta i} \leq \frac{t_{1}}{2} \\
-\frac{t_{1}}{2} \leq\left(\boldsymbol{\varepsilon}_{\mathrm{p}-1,1 / A B}+\mathbf{C D} \wedge \boldsymbol{\rho}_{1,1 / A B}\right) \cdot \mathbf{n}_{\theta i} \leq \frac{t_{1}}{2}
\end{array}\right\} \\
& \text { with }\left\{\begin{array}{l}
\mathbf{n}_{\theta i}=\cos \theta_{i} \cdot \mathbf{x}+\sin \theta_{i} \cdot \mathbf{y} \\
\theta_{i}=i \frac{\pi}{n}, 0 \leq i<n \text { and }(i, n) \in \mathbb{N}
\end{array}\right.
\end{aligned}
$$

The relations (16) define a bounded intersection of finite number of closed halfspaces whose boundaries are hyperplanes of $\mathbb{R}^{4}$ [34] intersection. In general, it can formalize the h-representation of a polytope called geometric polytope, noted $\mathfrak{D}_{1,1 / A B}^{g}$. $\mathfrak{D}_{1,1 / A B}^{g}$ represents the geometric polytope of the location of the surface 1,1 with respect to $A B$. This is 4-polytope in which two graphical representations are shown in 
Journal of Computing and Information Science in Engineering

Fig. 11. The Fig. 11a and the Fig. 11b represent two projections of 4-polytope respectively in $\left(\rho_{x}, \varepsilon_{P, y}\right)$ and in $\left(\rho_{y}, \varepsilon_{P, x}\right)$.

The orthogonality of the housing axis 1,1 with respect to the datum $A$ can be characterized by a geometric polytope $\mathfrak{D}_{1,1 / A}^{g}$. The axis of the cylindrical surface of substitution 1,1 is within a tolerance zone $T Z . T Z$ is a cylinder of diameter $\varnothing t_{2}$ orthogonal to the plane $A$. To ensure that the axis of the surface 1,1 , is within the orientation tolerance zone $T Z$, the orthogonal distance to the axis $\mathbf{y}$ between the points $C$ and $D$ must be less than the dimension of the tolerance zone:

$-t_{2} \leq\left(\boldsymbol{\varepsilon}_{C-1,1 / A}-\boldsymbol{\varepsilon}_{D-1,1 / A}\right) \cdot \mathbf{n}_{\theta i} \leq t_{2}$

with $\left\{\begin{array}{l}\mathbf{n}_{\theta i}=\cos \theta_{i} \cdot \mathbf{x}+\sin \theta_{i} \cdot \mathbf{y} \\ \theta_{i}=i \frac{\pi}{n}, 0 \leq i<n \text { and }(i, n) \in \mathbb{N}\end{array}\right.$

The relation (17) is expressed according to the rotation vector and the translation vector of the deviation of the point $\mathrm{P}$ middle of the bounded by line segment $B$ and C:

$$
\begin{aligned}
& -t_{2} \leq\left(\boldsymbol{\varepsilon}_{C-1,1 / A}-\boldsymbol{\varepsilon}_{D-1,1 / A}\right) \cdot \mathbf{n}_{\theta i} \leq t_{2} \\
& \text { with }\left\{\begin{array}{l}
\mathbf{n}_{\theta i}=\cos \theta_{i} \cdot \mathbf{x}+\sin \theta_{i} \cdot \mathbf{y} \\
\theta_{i}=i \frac{\pi}{n}, 0 \leq i<n \text { and }(i, n) \in \mathbb{N}
\end{array}\right.
\end{aligned}
$$

Relations (18) formalize the geometric polytope, noted $\mathfrak{D}_{1,1 / A}^{g}$, corresponding to the location of 1,1 with respect to the reference $A$. This is a 2-polytope whose graphical representation is given in Fig. 12 in $\left(\rho_{x}, \rho_{y}\right)$. 
Journal of Computing and Information Science in Engineering

Both specifications expressed on the axis 1,1 of the housing 1 must be respected simultaneously. The polytope characterizing the position of the axis 1,1 with respect to the datum reference frame $A B$ is the polytope resulting from the intersection between the polytopes $\mathfrak{D}_{1,1 / A B}^{g}$ and $\mathfrak{D}_{1,1 / A}^{g}$. This is a 4-polytope in which two graphical representations are shown in Fig. 13. Fig. 13a and Fig. 13b represent the projections of the 4-polytope in $\left(\rho_{x}, \varepsilon_{P, y}\right)$ and $\left(\rho_{y}, \varepsilon_{P, x}\right)$.

In a similar manner to the location and orthogonality specifications of the axis 1,1 of the housing 1 , the geometric polytope characterizing the position of the axis 2,1 with respect to the datum reference frame $C D$ result from the intersection of the polytopes $\mathfrak{D}_{2,1 / C D}^{\mathrm{g}}$ and $\mathfrak{D}_{2,1 / C}^{\mathrm{g}}$. This is 4-polytope in which two graphical representations are shown in Fig. 14. Fig. 14a and Fig. 14b represent the projections of 4-polytope in $\left(\rho_{x}, \varepsilon_{P, y}\right)$ and $\left(\rho_{y}, \varepsilon_{P, x}\right)$.

\subsection{Transfer of contact deviations between the parts}

Previously geometric deviations of parts were formalised by polytopes. In the following, deviations due to mobility and clearance in the joints will be formalised by polytopes. The joint cover/housing is composed of three elementary joints: a plane pair contact and two ball and cylinder pair contacts. To model the full joint, each of three joints is modelled by a contact polytope.

The planar pair contact between the plane 1,2 cover 2 and the plane 2,2 the housing 2 is without clearance (Fig. 6). The relation (19) characterised the contact without clearance on the whole contour of the contact surface: 


$$
\left\{\begin{array}{l}
\boldsymbol{\varepsilon}_{\mathrm{p}-1,2 / 2,2} \cdot \mathbf{z}=0 \\
\boldsymbol{\rho}_{1,2 / 2,2} \cdot \mathbf{x}=0 \\
\boldsymbol{\rho}_{1,2 / 2,2} \cdot \mathbf{y}=0
\end{array}\right\}
$$

Relations (20) characterize the limits of displacements between surfaces 1,3 and 2,3 and between surfaces 1,4 and 2,4 of the both ball and cylinder pair contacts (Fig. 6):

$$
\left\{\begin{array}{l}
-\frac{J}{2} \leq \boldsymbol{\varepsilon}_{\mathrm{E}-1,3 / 2,3} \cdot \mathbf{n}_{\theta i} \leq \frac{J}{2} \\
-\frac{J}{2} \leq \boldsymbol{\varepsilon}_{\mathrm{F}-1,4 / 2,4} \cdot \mathbf{n}_{\theta i} \leq \frac{J}{2}
\end{array}\right\}
$$

with $\left\{\begin{array}{l}\mathbf{n}_{\theta i}=\cos \theta_{i} \cdot \mathbf{x}+\sin \theta_{i} \cdot \mathbf{y} \\ \theta_{i}=i \frac{\pi}{n}, 0 \leq i<n \text { and }(i, n) \in \mathbb{N}\end{array}\right.$

The maximum clearance corresponds to cases where the diameters of the shafts are smaller and diameters of holes are larger:

$$
J=D_{\text {max_hole }}-D_{\text {min_pin }}=t_{5}+t_{6}
$$

Relation (20) is expressed according to the rotation vector and the translation vector of the deviation of the point $P$ :

$$
\begin{aligned}
& \left\{\begin{array}{l}
-\frac{J}{2} \leq\left(\mathbf{E P} \wedge \boldsymbol{\rho}_{1,3 / 2,3}\right) \cdot \mathbf{n}_{\theta i} \leq \frac{J}{2} \\
-\frac{J}{2} \leq\left(\mathbf{F P} \wedge \boldsymbol{\rho}_{1,4 / 2,4}\right) \cdot \mathbf{n}_{\theta i} \leq \frac{J}{2}
\end{array}\right\} \\
& \text { with }\left\{\begin{array}{l}
\mathbf{n}_{\theta i}=\cos \theta_{i} \cdot \mathbf{x}+\sin \theta_{i} \cdot \mathbf{y} \\
\theta_{i}=i \frac{\pi}{n}, 0 \leq i<n \text { and }(i, n) \in \mathbb{N}
\end{array}\right.
\end{aligned}
$$

The polytope of contact characterising the relative position between the housing 1 and the cover 2 results of the intersection between the polytopes defined in (19) and 
Journal of Computing and Information Science in Engineering

(22). This polytope called $\mathfrak{D}_{A B / C D}^{C}$ is a 3-polytope which a projection in $\left(\varepsilon_{\mathrm{P}, x}, \varepsilon_{\mathrm{P}, y}\right)$ is given in Fig. 15.

\subsection{Full condition}

Geometrical deviations in parts and in contacts were respectively determined in sections 4.2 and 4.3. The relative position between the bore of the housing and the cover will be characterized by the calculated polytope defined by the following Minkowski sum:

$\mathfrak{D}_{1,1 / 2,1}=\mathfrak{D}_{1,1 / A B}^{\mathrm{g}}+\mathfrak{D}_{A B / C D}^{\mathrm{c}}+\mathfrak{D}_{C D / 2,1}^{\mathrm{c}}$

The functional polytope $\mathfrak{D}_{1,1 / 2,1}^{\mathfrak{l}}$ characterizing the straightness between the bore axis of the cover and the housing is defined by the following relation:

$\mathfrak{D}_{1,1 / 2,1}^{\mathrm{f}}=\mathfrak{D}_{1,1 / 2 \mathrm{~T}}^{\mathrm{f}}+\mathfrak{D}_{Z T / 2,1}^{\mathrm{f}}$

The Fig. 16 illustrates the result of the sum of Minkowski (24) characterizing the polytope calculated $\mathfrak{D}_{1,1 / 2,1}$ in $\left(\rho_{x}, \varepsilon_{P, y}\right)$. On this same figure, the functional polytope $\mathfrak{D}_{1,1 / 2,1}^{f}$ is shown. To characterize the straightness, the polytope must be included in calculating the functional polytope.

If the calculated polytope is included in the functional polytope, the straightness of the two bores is inferior to the value defined in the polytope functional. To determinate the value of straightness of the two bores is necessary to minimize the size of the functional polytope. When the size of the functional polytope is minimal, each 
vertex of the calculated polytope must be included into each half-spaces of the functional polytope. The straightness is defined by these inequalities:

$$
\begin{aligned}
& r \geq \frac{E_{C}}{E_{C}+L_{B C}}\left[\frac{\mathrm{t}_{1}}{2}+\frac{\mathrm{t}_{3}}{2}+\mathrm{t}_{4} \cdot \frac{\mathrm{L}_{B C}}{\mathrm{E}_{C}}+k \cdot\left(\mathrm{t}_{5}+\mathrm{t}_{6}\right)\right] \\
& r \geq \frac{E_{C}}{E_{C}+E_{H}+L_{B C}}\left[\frac{\mathrm{t}_{1}}{2}+\frac{\mathrm{t}_{3}}{2}+\mathrm{t}_{4} \cdot \frac{\mathrm{L}_{B C}+E_{H}}{\mathrm{E}_{C}}+k \cdot\left(\mathrm{t}_{5}+\mathrm{t}_{6}\right)\right] \quad(b) \\
& r \geq \frac{E_{H}}{E_{H}+L_{B C}}\left[\frac{\mathrm{t}_{1}}{2}+\frac{\mathrm{t}_{3}}{2}+\mathrm{t}_{2} \cdot \frac{\mathrm{L}_{B C}}{\mathrm{E}_{H}}+k \cdot\left(\mathrm{t}_{5}+\mathrm{t}_{6}\right)\right] \\
& r \geq \frac{E_{H}}{E_{H}+E_{C}+L_{B C}}\left[\frac{\mathrm{t}_{1}}{2}+\frac{\mathrm{t}_{3}}{2}+\mathrm{t}_{2} \cdot \frac{\mathrm{L}_{B C}+E_{C}}{\mathrm{E}_{H}}+k \cdot\left(\mathrm{t}_{5}+\mathrm{t}_{6}\right)\right](d)
\end{aligned}
$$

In these four inequalities (25), $r$ is greater into the relation (a) than into the relation (b) and $r$ is greater into the relation (c) than into the relation (d).

$$
\begin{aligned}
& r \geq \frac{E_{C}}{E_{C}+L_{B C}}\left[\frac{\mathrm{t}_{1}}{2}+\frac{\mathrm{t}_{3}}{2}+\mathrm{t}_{4} \cdot \frac{\mathrm{L}_{\mathrm{BC}}}{\mathrm{E}_{C}}+k \cdot\left(\mathrm{t}_{5}+\mathrm{t}_{6}\right)\right] \\
& r \geq \frac{E_{H}}{E_{H}+L_{B C}}\left[\frac{\mathrm{t}_{1}}{2}+\frac{\mathrm{t}_{3}}{2}+\mathrm{t}_{2} \cdot \frac{\mathrm{L}_{\mathrm{BC}}}{\mathrm{E}_{\mathrm{H}}}+k \cdot\left(\mathrm{t}_{5}+\mathrm{t}_{6}\right)\right]
\end{aligned}
$$

With this method, the straightness value defined into the relation (26) is the same as the previous method defined into relation (13).

\section{Conclusion}

Both methods integrate the influence of the geometrical defects of location and orientation, and the mobility allowed by the clearance in the joints. But they consider directly the situation in the worst case defined by the minimal material condition in the joints and the maximal material condition in the bearings. 
Journal of Computing and Information Science in Engineering

For primary planar joint, the analysis line method considers that the datum reference of one part remains in the tolerance zone of the support part. The polytope method neglects the flatness of planes in contact for superimposing the datum reference and the support surface. These two ways of expressing the assumptions lead to the same behaviour.

This study shows that geometric models are identical, and that the analysis lines means studying the polytope in particular directions. The polytope is more complex, but gives a global view of all degrees of freedom. The method of analysis line is more direct, but requires considering each requirement in several directions by discretization of the ending surface.

Analysis line method directly gives the result as a linear formula of tolerances of influential parts, which subsequently allows to globally optimize the whole system of inequalities to maximize tolerances.

Both methods require a lot of rigor and expertise. A specific tool in CAD system is necessary. The setting-up of parts and functional requirements are described by the designer. The tolerance analysis application collects geometric data and the influential annotations. The calculations can be fully automated to display the result in the form of the numerical value of the result to be compared with the limit value of the requirement. 
Journal of Computing and Information Science in Engineering

\section{NOMENCLATURE}
$d\left(A_{B}, \mathbf{x}\right) \quad$ maximum displacement of point $A$ of the part $B$ in $\mathbf{x}$ direction
$\rho_{x} \quad$ rotation along $x$ axis
$\varepsilon_{P, y} \quad$ Translation along y axis at point B
$\boldsymbol{\rho}_{i, j / u, v} \quad$ rotation vector of the surface $\mathrm{j}$ of the part $\mathrm{i}$ with respect to the surface $\mathrm{v}$ of the part $u$
$\boldsymbol{\varepsilon}_{\mathrm{B}-i, j / u, v} \quad$ rotation vector of the surface $j$ of the part $\mathrm{i}$ with respect to the surface $v$ of the part $u$ at point $B$

$\mathfrak{D}_{i, j / i, k}^{g}$ tla geometric polytope of the surface $\mathrm{j}$ of the part $\mathrm{i}$ with respect to the surface $k$ of the part $\mathrm{i}$

$\mathfrak{D}_{i, j / u, v}^{c} \operatorname{Re} \quad$ contact polytope of the surface $\mathrm{j}$ of the part $\mathrm{i}$ with respect to the surface $\mathrm{v}$ of the part $\mathrm{u}$

$\mathfrak{D}_{i, j / u, v}^{f} T \quad$ functional polytope of the surface $\mathrm{j}$ of the part $\mathrm{i}$ with respect to the surface $v$ of the part $u$ 
Journal of Computing and Information Science in Engineering

\section{REFERENCES}

[1] Salomons, O.W., Haalboom, F. J., Jonge Poerink, H. J., van Slooten, F., Van Houten, F. J. A. M., and Kals, H.J. J., 1996, "A computer aided tolerancing tool i: Tolerancing specification," Computers in Industry, 31(2), pp. 161-174. DOI:10.1016/01663615(96)00046-2

[2] Salomons, O.W., Haalboom, F. J., Jonge Poerink, H. J., van Slooten, F. , Van Houten, F. J. A. M., and Kals, H. J. J., 1996, "A computer aided tolerancing tool ii: Tolerancing analysis," Computers in Industry, 31(2), pp. 175-186. DOI: 10.1016/01663615(96)00047-4

[3] Chase, K. W., 1999, "Tolerance allocation methods for designers," ADCATS Report, 99(6), Brigham Young University

[4] Islam, M.N., 2004, "Functional dimensioning and tolerancing software for concurrent engineering applications," Computers in Industry, 54(2), pp. 169-190. DOI:

10.1016/j.cad.2006.05.005

[5] Anselmetti, B., 2006, "Generation of functional tolerancing based on positioning features," Computer-Aided Design, 38(8), pp. 902-919. DOI: 10.1016/j.cad.2006.05.005

[6] Johannesson, H., and Soderberg, R., 2000, "Structure and matrix models for tolerance analysis from configuration to detail design," Research in engineering design, 12(2), pp. 112-125. DOI: 10.1007/s001630050027

[7] Ballot, E., and Bourdet, P., 1995, "Geometrical behavior laws for computer aided tolerancing," Proc. 4th CIRP Seminar on Computer Aided Tolerancing, Tokyo, Japan, pp. 143-153.

[8] Mehdi-Souzani, C., and Anselmetti, B., "Integration of automatic functional tolerancing into the design process," 2008, Proc. IDMME - Virtual Concept, Beijing, China.

[9] Nigam, S.D., and Turner, J.U., 1995, "Review of statistical approaches to tolerance analysis," Computer Aided Design, 27(1), pp. 6-15. DOI: 10.1016/0010-4485(95)90748-5

[10] Shan, A., and Roth, R.N., 2003, "Genetic algorithms in statistical tolerancing," J. Mathematical and Computer Modeling, 38(11-13), pp. 1427-1436. DOI: 10.1016/S08957177(03)90146-4

[11] Kuo, C.,H., and Tsai, J.C., 2010, "An analytical computation method for statistical tolerance analysis of assemblies with truncated normal mean shift," International 
Journal of Computing and Information Science in Engineering

Journal of Production Research, 49(7), pp. 1937-1955. DOI:

10.1080/00207541003639634

[12] Skowronski, V.J., and Turner, J.U., 1997, "Using Monte Carlo variance reduction in statistical tolerance synthesis," Comput-Aided Design, 29(1), pp. 63-69. DOI:

10.1016/S0010-4485(96)00050-4

[13]. Lin, C., Huang, W., Jeng, M., and Doong, J.L., 1997, "Study of an assembly tolerance allocation model based on Monte Carlo simulation," Journal of Materials Processing Technology, 70(1), pp. 9-16.DOI:10.1016/s0924-0136(97)00034-4

[14] Shah, J.J., Ameta, G., Shen, Z., and Davidson, J.K., 2007, "Navigating the tolerance analysis maze," Computer-Aided Design and Applications, 4(5), pp. 705-718. DOI: 10.1080/16864360.2007.10738504

[15] Giordano, M., Pairel, E., and Samper, S., 1999, "Mathematical representation of tolerance zones," Proc. 6th CIRP Seminar on Computer Aided Tolerancing, University of Twente, Enschede, The Netherland, Global Consistency of Tolerances, Kluwer Academic Publishers Ed.,pp. 177-186. ISBN: 978-94-017-1705-2

[16] Davidson, J.K., Mujezinovic, A., and Shah, J.J., 2002, "A new mathematical model for geometric tolerances as applied to round faces," ASME Transactions on Journal of Mechanical Design, 124(4), pp. 609-622. DOI:10.1115/1.1497362

[17] Roy, U., and Li, B., 1999, "Representation and interpretation of geometric tolerances for polyhedral objects. II.: Size, orientation and position tolerances," Computer-Aided Design, 31(4), pp. 273-285. DOI: 10.1016/S0010-4485(99)00028-7

[18] Pierre, L., Teissandier, D., and Nadeau, J.P., 2009, "Integration of thermomechanical strains into tolerancing analysis," International Journal on Interactive Design and Manufacturing, 3(4), pp. 247-263. DOI: 10.1007/s12008-009-0058-8

[19] Pierre, L., Teissandier, D., and Nadeau, J.P., 2014, "Variational tolerancing analysis taking thermomechanical strains into account: Application to a high pressure turbine," Mechanism and Machine Theory, 74, pp. 82-101. DOI:

10.1016/j.mechmachtheory.2013.11.014

[20] Clozel, P., and Rance, P. A., 2010, "MECAmaster: a tool for assembly simulation from early design, industrial approach," Geometric Tolerancing of Products, Wiley-ISTE ed., 241-273. ISBN: 978-1-84821-118-6

[21] CETOL.6 http://www.sigmetrix.com/.

[22] DCS, 2012, "Variation Analysis and Tolerance Analysis," www.3dcs.com. 
Journal of Computing and Information Science in Engineering

[23] Falgarone, H., and Chevassus, N., 2004, "An innovative Design Method and Tool for Structural and Functional Analysis," Proc. 14th CIRP Design Seminar, Cairo, Egypt.

[24] Loof, J., Hermansson, T., and Sodderberg, R., 2005, "An efficient solution to the discrete Least Cost tolerance allocation problem with general loss functions," Model for Computer Aided tolerancing in design and manufacturing, Springer ed, pp 115-124. ISBN: 978-1-4020-5438-9

[25] Kurlarni, S.V., and Garg, T.K., 1987, "Allocation of tolerances to the components of an assembly for minimum cost," International Journal of Mechanical Engineering, 67(6), pp. 126-129.

[26] Anselmetti, B., 2010, "Part optimization and tolerances synthesis," International Journal of Advanced Manufacturing Technology, 48(9-12), pp. 1221-1237. DOI: 10.1007/s00170-009-2355-6

[27] Khanafer, M., and Desrochers, A., and Laperrière, L., 2007, "Tolerancing assistance methodology in a product life cycle perspective," Proc. 18th IASTED International Conference: modelling and simulation, ACTA Press Ed., Anaheim, CA, USA, pp. 514-520.

[28] Benichou, S., and Anselmetti, B., 2011, "Thermal dilatation in functional tolerancing," Mechanism and Machine Theory, 46(11), pp. 1575-1587. DOI: 10.1016/j.mechmachtheory.2011.06.009

[29] Fleming, A., 1988, "Geometric relationships between toleranced features," Artificial Intelligence, 37(1-3), pp. 403-412. DOI: 10.1016/0004-3702(88)90062-8

[30] ISO1101:2013, 2013, Geometrical Product Specifications (GPS), Geometrical tolerancing, Tolerances of form, orientation, location and run-out.

[31] Fleming, A.D., 1987, "Analysis of Uncertainties and Geometric Tolerances in Assemblies of Parts," PhD thesis of University of Edinburgh.

[32] Srinivasan, V., 1993, "Role of Sweeps in Tolerancing Semantics", Proc. International Forum on Dimensional Tolerancing and Metrology, (1993), CRTD, 27, pp. 69-78.

[33] Giordano, M., and Duret, D., 1993, "Clearance Space and Deviation Space," Proc. 3rd CIRP seminar on Computer Aided Tolerancing, ENS Cachan, France, PYC Ed., pp. 179196.

[34] Teissandier, D., and Delos, V., 1999, "Operations on polytopes: application to tolerance analysis," Proc. 6th CIRP Seminar on Computer Aided Tolerancing, University 
Journal of Computing and Information Science in Engineering

of Twente, Enschede, The Netherland, Global Consistency of Tolerances, Kluwer Academic Publishers Ed., pp. 425-433.

[35] Wu, Y., Shah, J.J., and Davidson, J.K., 2003, "Improvements to algorithms for computing the Minkowski sum of 3-polytopes," Computer-Aided Design, 35(13), pp. 1181-1192. DOI: 10.1016/S0010-4485(03)00023-X

[36] Teissandier, D., and Delos ,V. , 2011, "Algorithm to calculate the Minkowski sums of 3-polytopes based on normal fans," Computer-Aided Design, 43(12), pp. 1567-1576. DOI: 10.1016/j.cad.2011.06.016

[37] Anselmetti, B., Chavanne, R., Yang, J-Y., and Anwer, N., 2010, "Quick GPS: A new CAT system for single-part tolerancing," Computer-Aided Design, 42(9), pp. 768-780. DOI: 10.1016/j.cad.2010.04.006

[38] Chavanne, R., 2011, "Contribution au tolérancement fonctionnel 3D des mécanismes complexes : Synthèse des spécifications et Analyse de tolerances," PhD thesis of ENS Cachan. 
Journal of Computing and Information Science in Engineering

\section{Figure Captions List}

Fig. 1 Mechanism

Fig. 2 Setting-up table of the cover on the housing

Fig. $3 \quad$ Link inside cover

Fig. $4 \quad$ ISO tolerancing of both parts

Fig. 5 Assembling requirement of the shaft

Fig. $6 \quad$ Main specifications

Fig. 7 Discretization in 8 analysis directions

Fig. 8 Straightness between two different parts

Fig. 9 Influence of the cover

Fig. 10 Influence of junction housing/cover

Fig. 11 Geometric polytope $\mathfrak{D}_{1,1 / A B}^{\mathrm{g}}$

Fig. $12 \quad$ Geometric polytope $\mathfrak{D}_{1,1 / A}^{g}$

Fig. $13 \quad$ Global geometric polytope $\mathfrak{D}_{1,1 / A B}^{\mathrm{g}}$

Fig. $14 \quad$ Geometric polytope $\mathfrak{D}_{2,1 / C D}^{\mathrm{g}}$

Fig. 15 Polytope of contact $\mathfrak{D}_{A B / C D}^{C}$

Fig. 16 Inclusion of the calculated polytope $\mathfrak{D}_{1,1 / 2,1}$ in the functional polytope $\mathfrak{D}_{1,1 / 2,1}^{\mathrm{f}}$ 
Journal of Computing and Information Science in Engineering

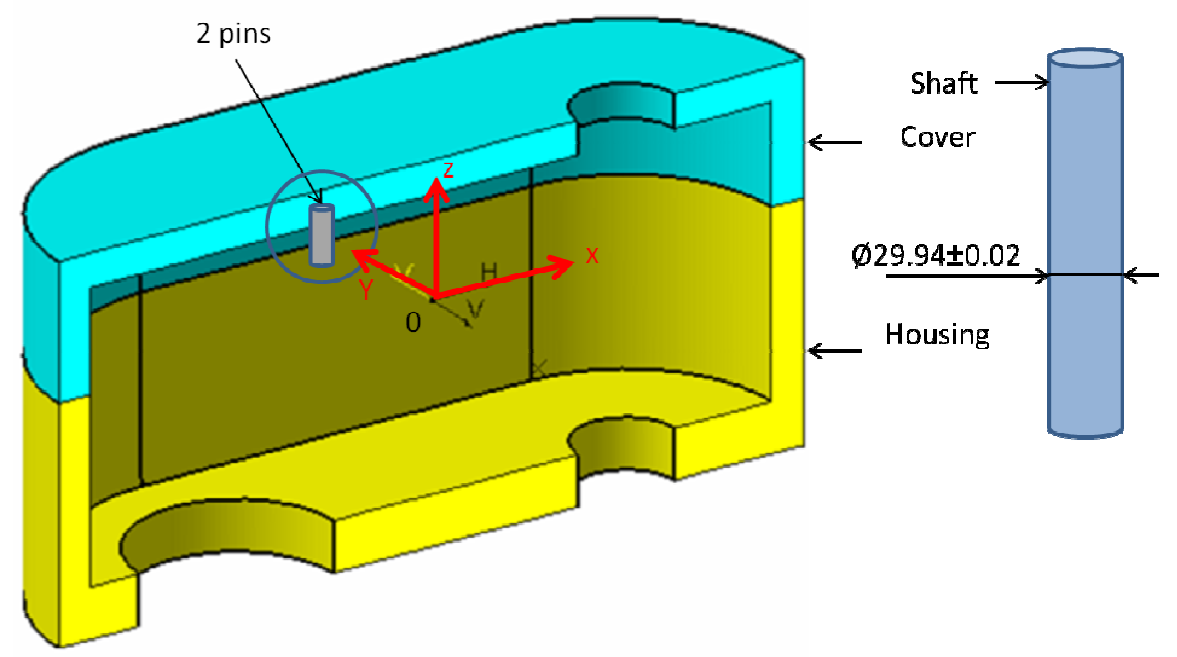

Fig. 1. Mechanism 
Journal of Computing and Information Science in Engineering

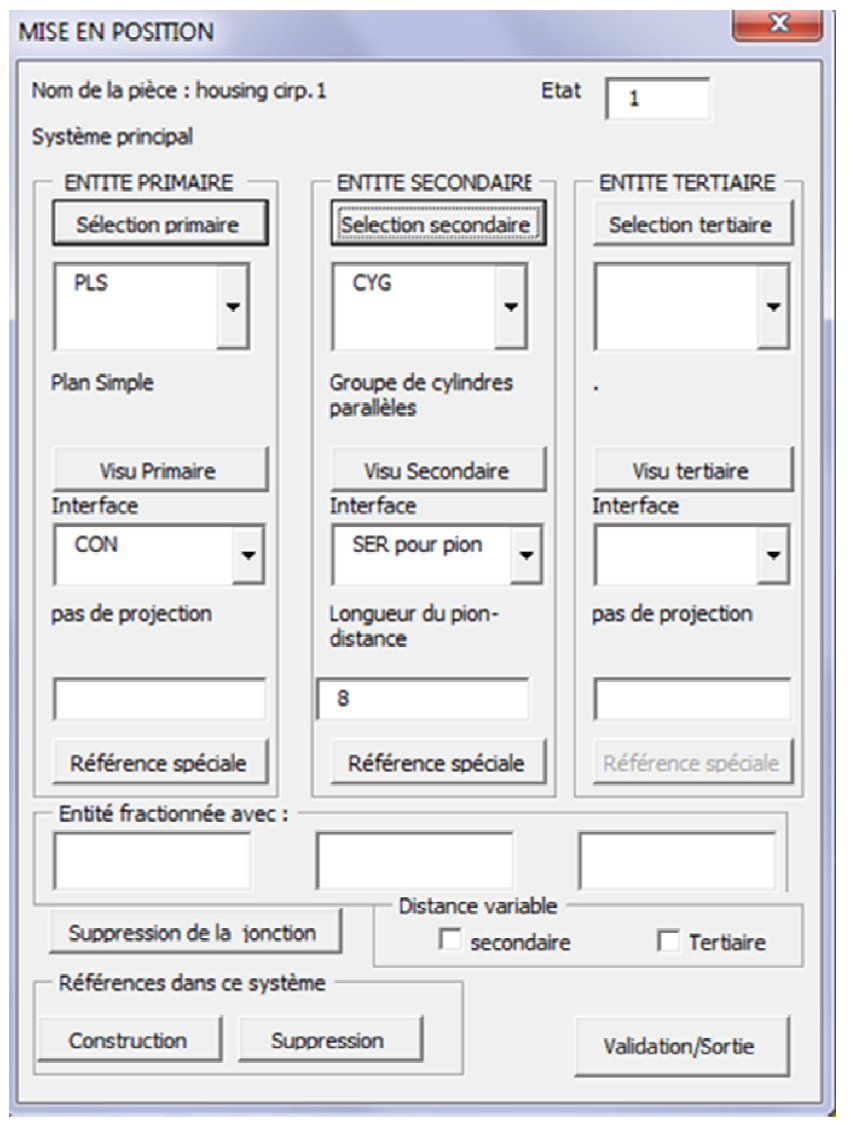

Fig. 2. Setting-up table of the cover on the housing 
Journal of Computing and Information Science in Engineering

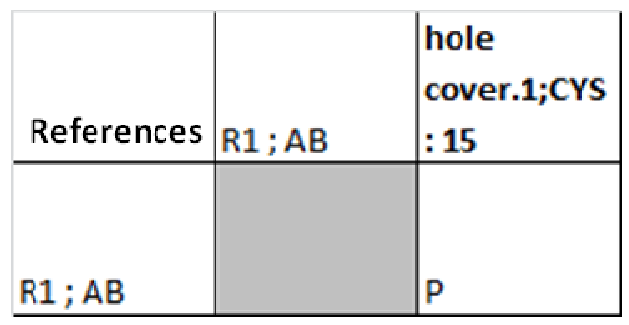

Fig. 3. Link inside cover 
Journal of Computing and Information Science in Engineering

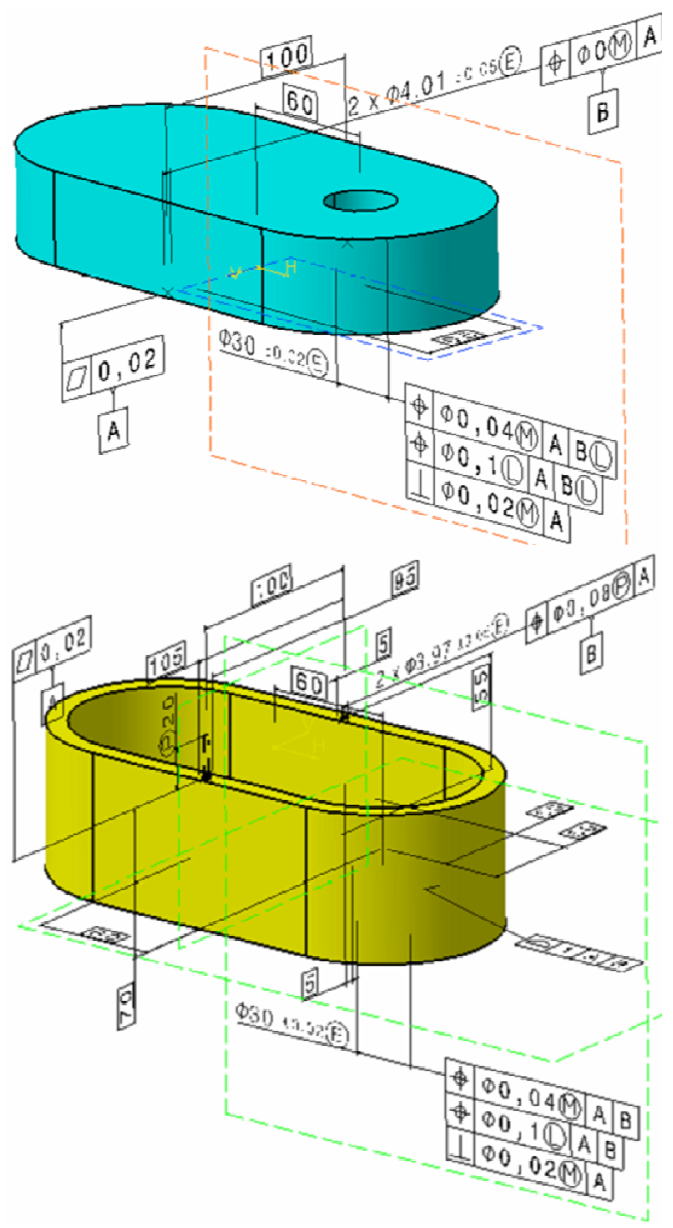

Fig. 4. ISO tolerancing of both parts 
Journal of Computing and Information Science in Engineering

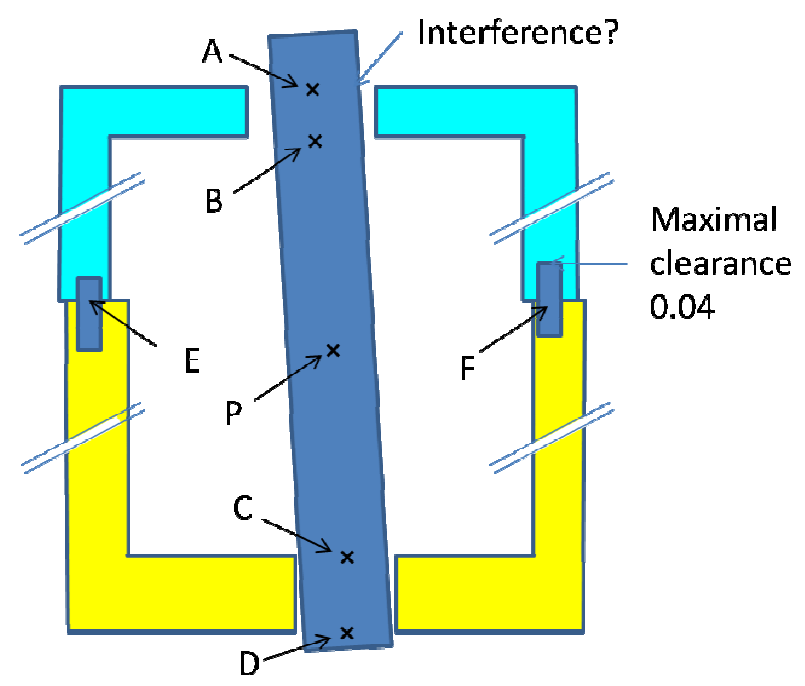

Fig. 5. Assembling requirement of the shaft 
Journal of Computing and Information Science in Engineering

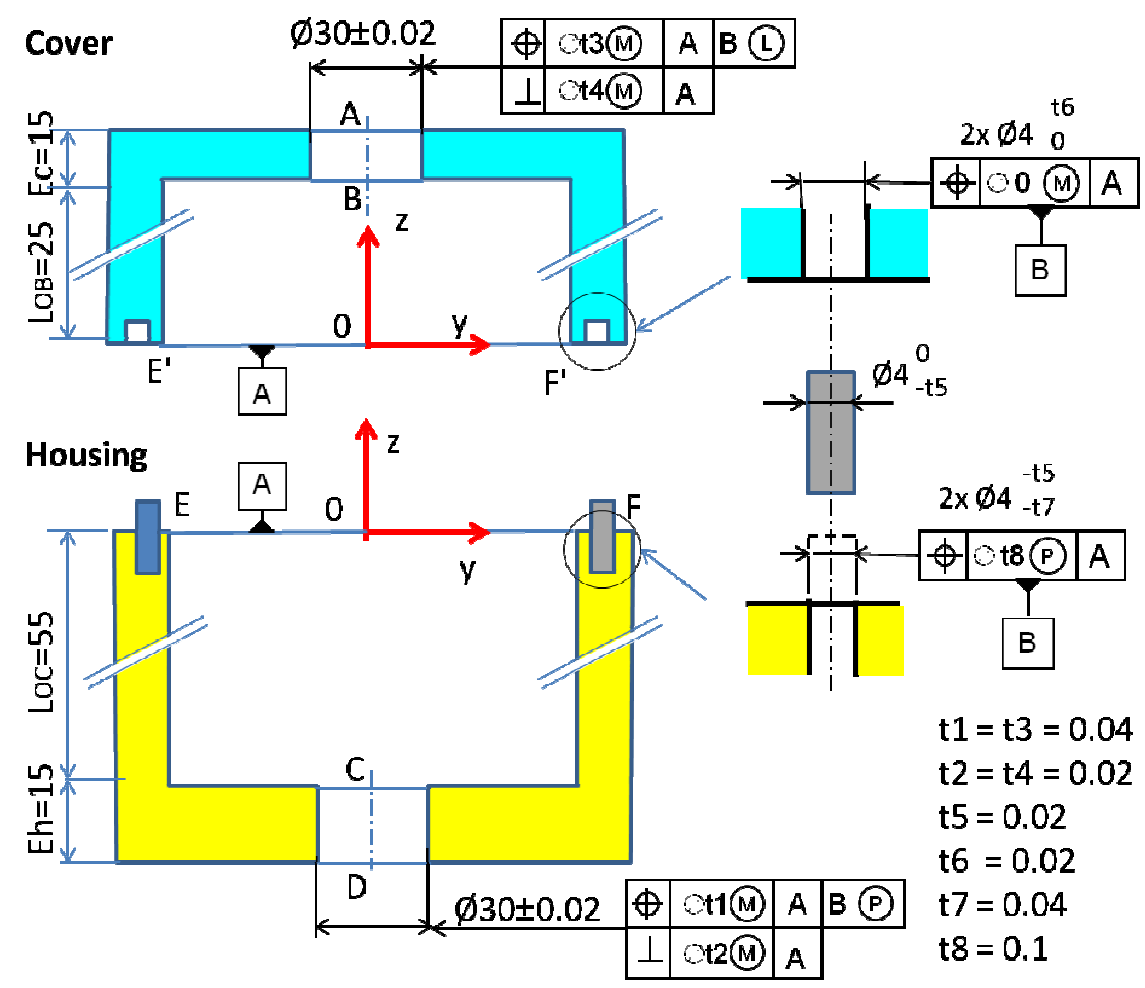

Fig. 6. Main specifications 
Journal of Computing and Information Science in Engineering

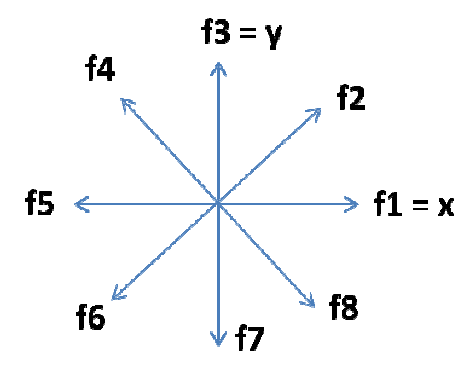

Fig. 7. Discretization in 8 analysis directions 
Journal of Computing and Information Science in Engineering

(a)

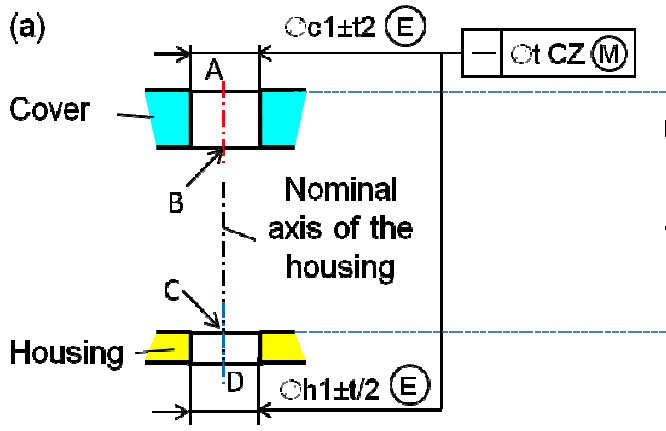

(c) $\mathrm{ri}$

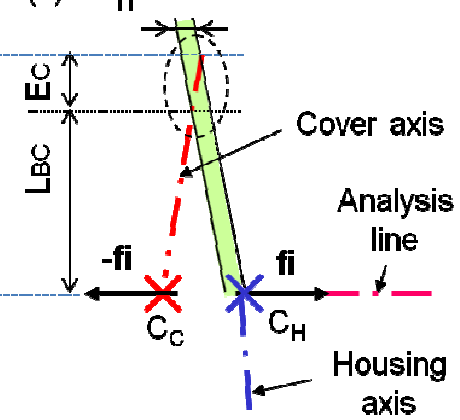

(b)

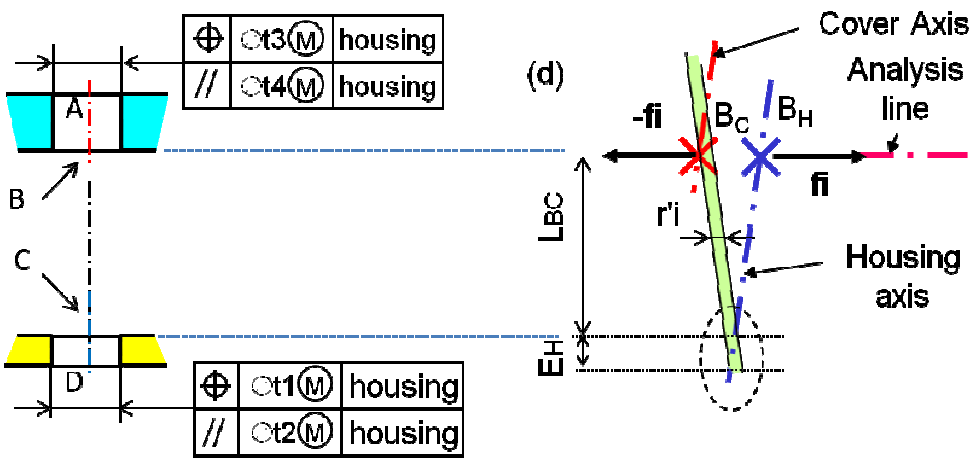

Fig. 8. Straightness between two different parts 
Journal of Computing and Information Science in Engineering

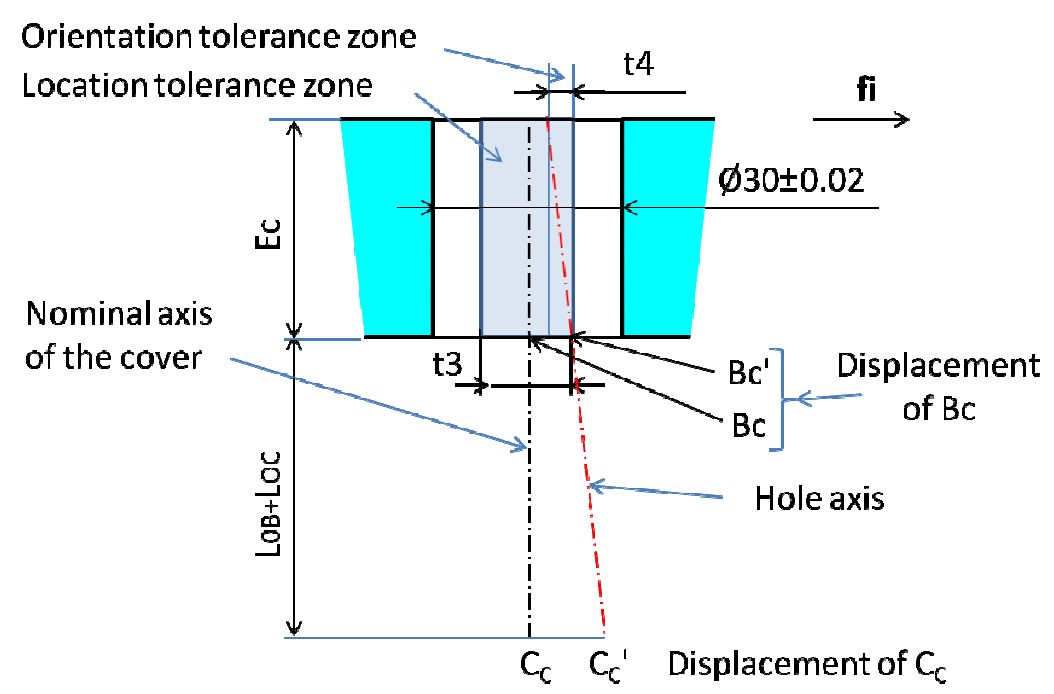

Fig. 9. Influence of the cover 
Journal of Computing and Information Science in Engineering

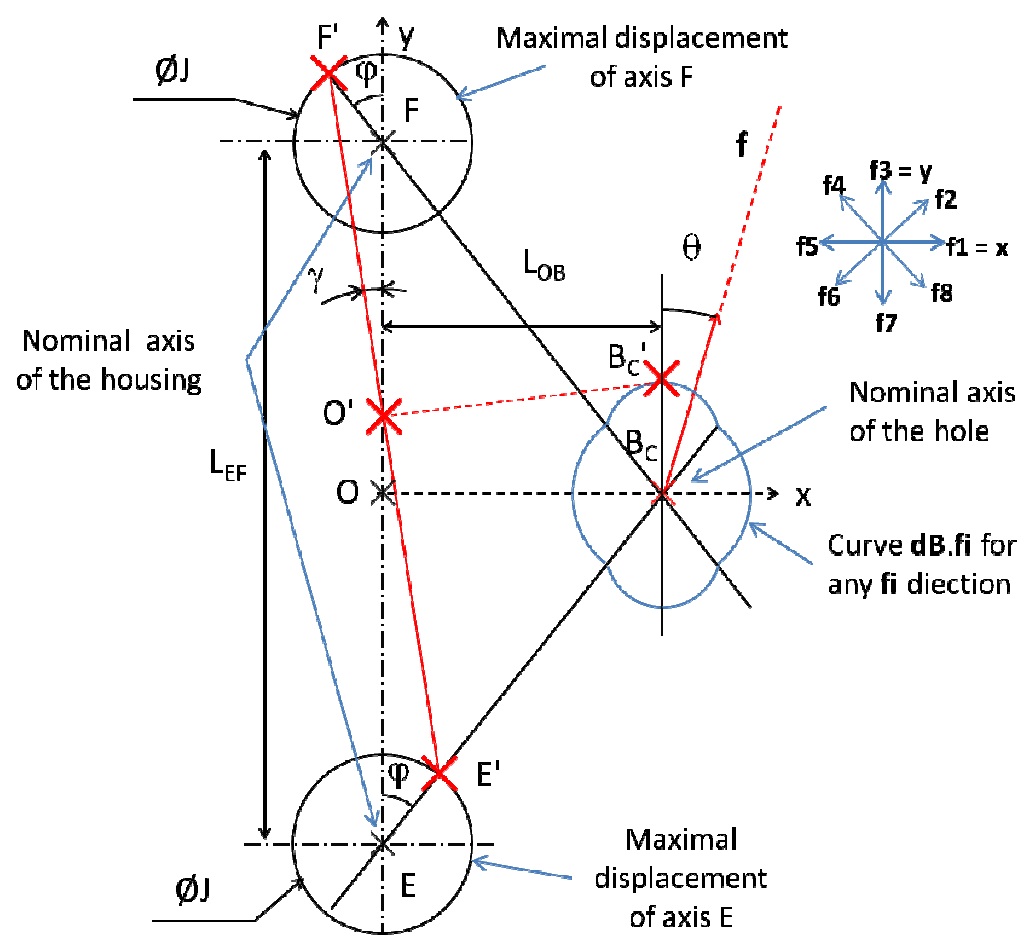

Fig. 10. Influence of junction housing/cover 
Journal of Computing and Information Science in Engineering

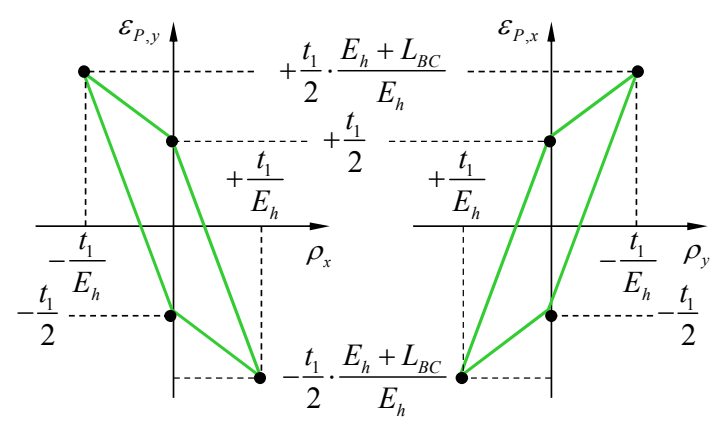

Fig. 11. Geometric polytope $\mathfrak{D}_{1,1 / A B}^{g}$ 
Journal of Computing and Information Science in Engineering

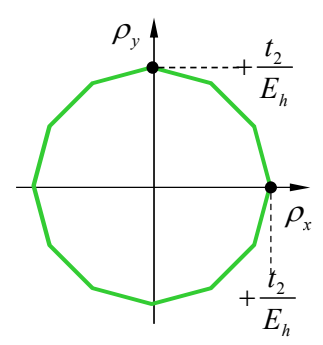

Fig. 12. Geometric polytope $\mathfrak{D}_{1,1 / A}^{\mathrm{g}}$ 
Journal of Computing and Information Science in Engineering

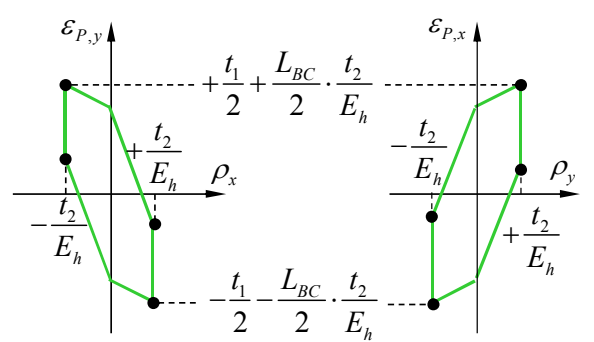

Fig. 13. Global geometric polytope $\mathfrak{D}_{1,1 / A B}^{g}$ 
Journal of Computing and Information Science in Engineering

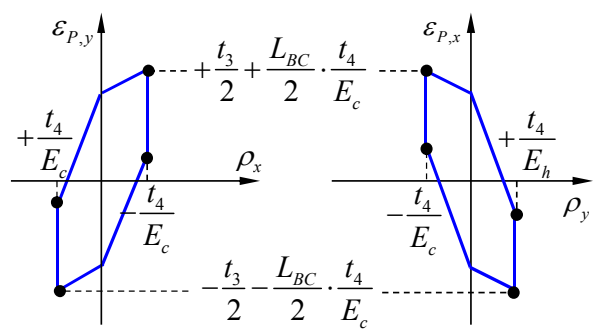

Fig. 14. Geometric polytope $\mathfrak{D}_{2,1 / C D}^{g}$ 
Journal of Computing and Information Science in Engineering

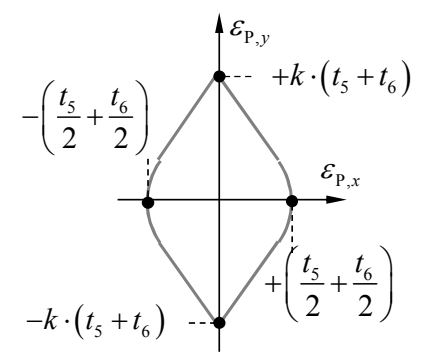

Fig. 15. Polytope of contact $\mathfrak{D}_{A B / C D}^{C}$ 
Journal of Computing and Information Science in Engineering

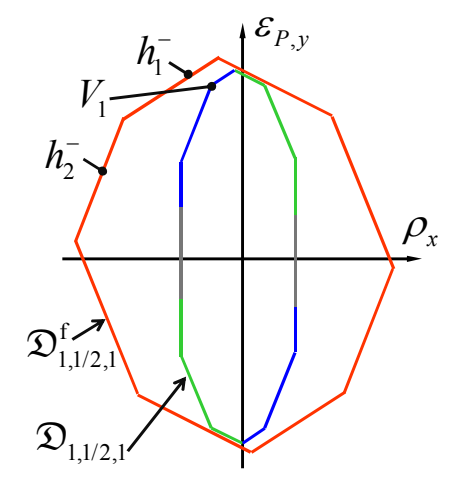

Fig. 16. Inclusion of the calculated polytope $\mathfrak{D}_{1,1 / 2,1}$ in the functional polytope $\mathfrak{D}_{1,1 / 2,1}^{\mathrm{f}}$ 